\title{
ATMOSPHERIC FLOWS IN LARGE WIND FARMS
}

IR.A. Verzijlbergh - DOI: https://doi.org/10.1051/epn/2021502

n Delft University of Technology, faculty of Technology, Policy and Management and Whiffle Weather Forecasting

As we are transitioning to an energy system based on renewable sources, the atmosphere is becoming one of our primary energy sources. Understanding atmospheric flows through wind farms has become an issue of large economic and societal concern.

wo characteristics of renewable energy sources make them difficult to integrate in our power system: variability and unpredictability. Variability refers to their fluctuating output in concert with weather systems. These fluctuations cover a range of time scales from years to sub-seconds. Unpredictability means that, unlike conventional power plants, the output of renewable energy sources cannot be predicted exactly; neither in the short-term (hours to days ahead) nor in a climatological sense in the long-term (over multiple years). The intermittency of renewable energy sources requires a certain amount of flexible resources like energy storage or back-up power plants to accompany the build-out of wind and solar farms. However, such technical solutions are costly and better knowledge of the atmospheric conditions that drive power production can avoid such investments to some extent. In this article we highlight some aspects related to modelling the flow and power production of the new generation of wind farms. These have rapidly become much larger, growing from $500 \mathrm{MW} 10$ years ago to $1500 \mathrm{MW}$ today and expected to reach the 4000 MW mark by 2025 . 

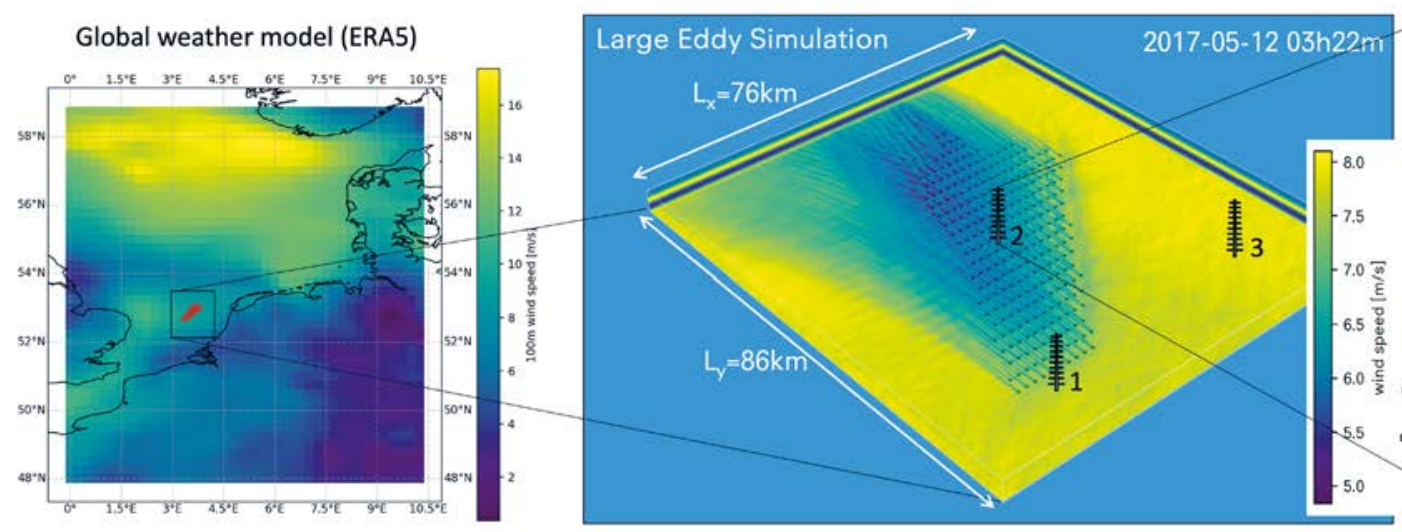

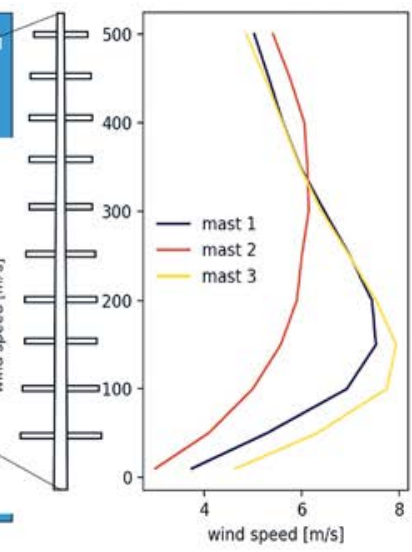

\section{Predicting the yield of wind farms}

The first step in modelling the expected production of a wind farm is to characterise the wind climate in absence of the wind farm, sometimes referred to as free-stream wind. To this end, local wind measurements are complemented with the output of numerical weather prediction models and micro-models to obtain a climatological "wind speed map" over the site. Especially for wind farms in complex environments (e.g. in forests, mountainous terrain or close to other wind farms), the wind speed can have strong local variations and the micro-model is an essential step.

The second important step is predicting how the wind turbines themselves interact with the flow inside the wind farm. Wind turbines have been designed with an important objective: extracting kinetic energy from the atmosphere and converting it to electrical energy. They are very efficient in doing this and so behind a wind turbine there is a wake region with a significant reduction in wind speed. Models of different complexity to predict wind farm wake losses exist, from simple engineering or analytical models to more complex computational fluid dynamics models. When applying these wake models, the free-stream wind as described above is used as input for the wake models. The large-scale flow is thus assumed to be independent of the flow inside the farm. However, this is becoming a problematic assumption, especially for the new generation of wind farms, which are becoming ever larger and built more closely together. One of the challenges in understanding wind farm flow physics is the coupling between the micro-scale (say, turbine level $\sim 100 \mathrm{~m}$ ) and the mesoscale (100 km or more) which is relevant for atmospheric processes [1].

\section{Large-Eddy Simulation}

Large-Eddy Simulation (LES) is a computational technique that has been successfully applied in both wind energy science and atmospheric physics. The essence is to numerically integrate the filtered conservation equations for momentum, mass, temperature and moisture on a grid that is fine enough to capture the largest part of the turbulent spectrum. In this way, turbulence is explicitly simulated, rather than parameterised. Atmospheric LES $\triangle$ FIG. 1: Left: snapshots of wind speed at $100 \mathrm{~m}$ height over the North Sea as modeled by ERA5 (the ECMWF $5^{\text {th }}$ Generation of the atmospheric reanalysis of the global climate covering the period from January 1950 to present). Middle: Large-Eddy Simulation driven by ERA5 of the planned IJmuiden Ver 4GW wind farm. Individual turbines are visualised with black dots. Right: Vertical profiles of wind speed for three virtual met-mast locations.

codes also include processes like radiation, cloud thermodynamics and the land- or sea-atmosphere interactions. Wind turbines can be represented as semi-permeable rotating disks that exert wind speed dependent forces on the flow. LES thus provides a unified framework to capture wind farm flows and windfarm atmosphere interactions in a single model. Its large computational costs have long been considered as a barrier for practical applications, but innovations in computer science, e.g. exploiting Graphics Processing Units, have moved LES to the frontier of models for the wind energy industry $[2,3,4]$.

V FIG. 2: Eight day time series of the modeled turbine power and wind speed in the IJmuiden Ver 4GW wind farm. Top: all individual turbine powers and turbine average power. Bottom: hub height wind speed at all turbines and free-stream wind speed.
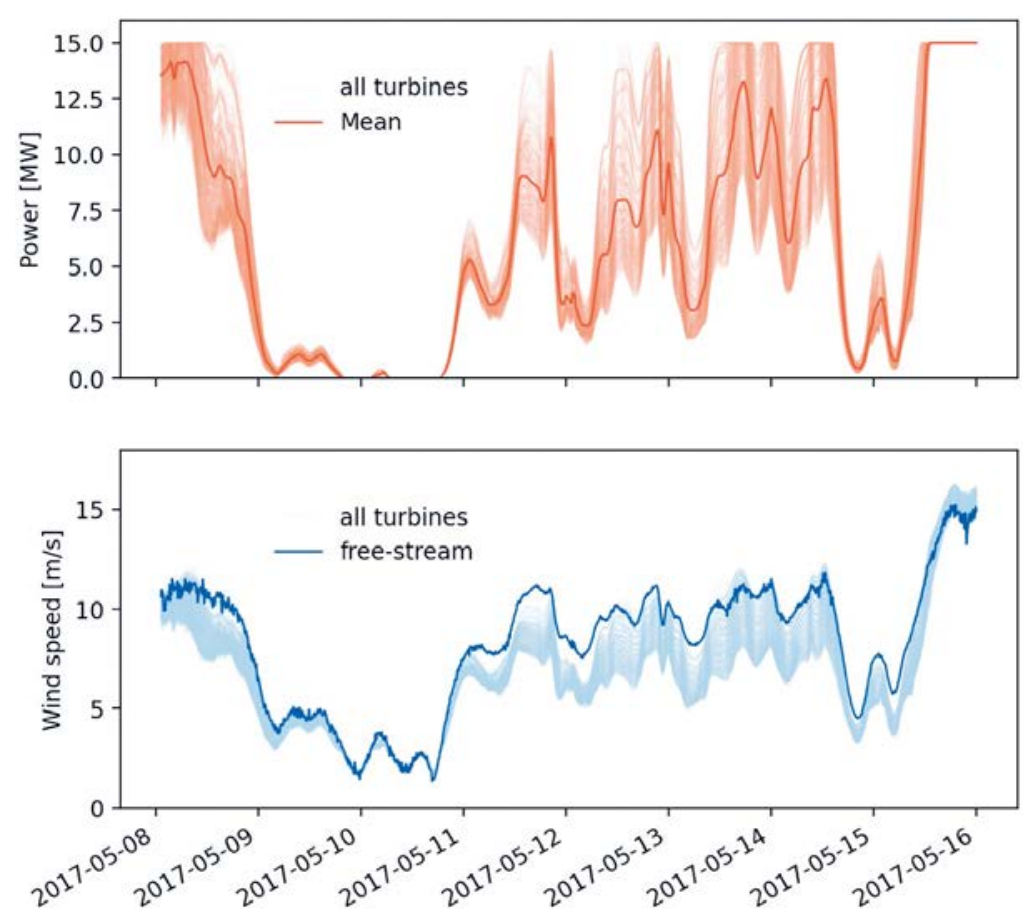

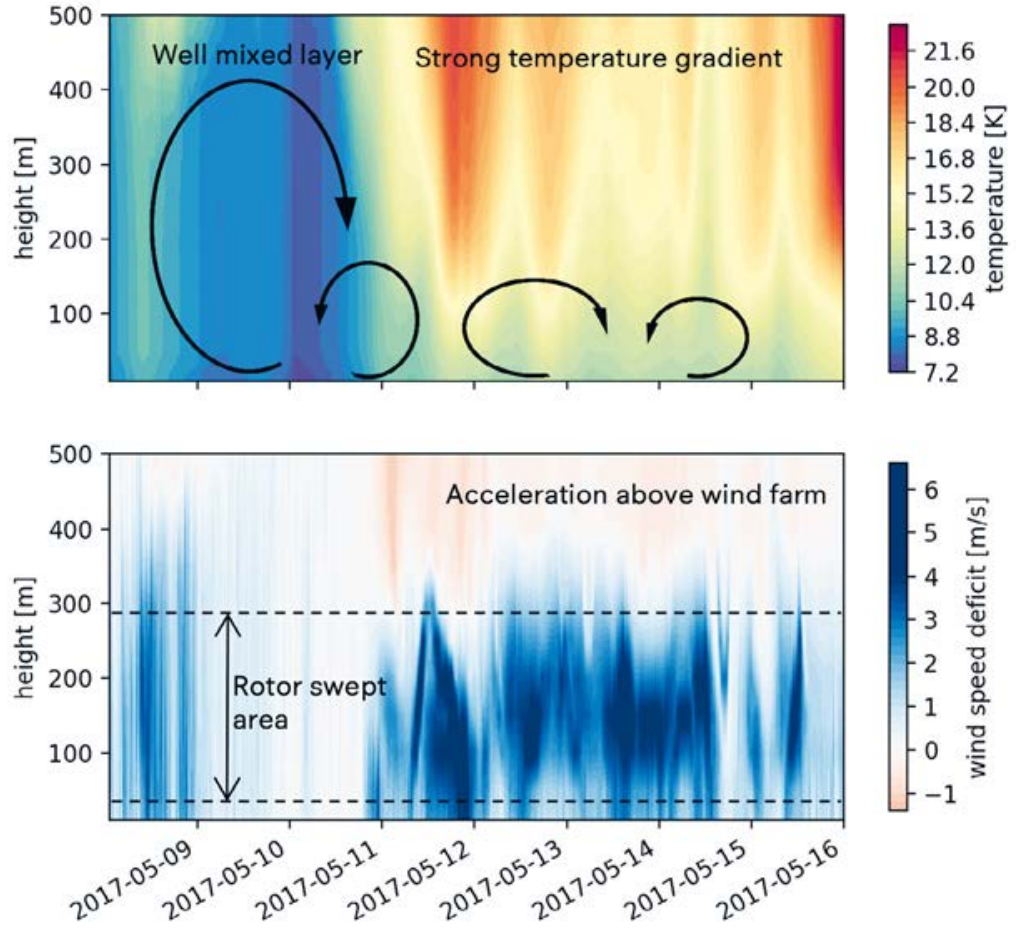

$\triangle$ FIG. 3: Temperature profile over time (top) and velocity deficit in the middle of the wind farm (bottom).

\section{Large wind farm physics at the frontier of flow modelling}

To illustrate some of the issues surrounding wind farm yield predictions, we present results of an atmospheric LES encompassing a large $\left(100 \mathrm{~km}^{2}\right)$ wind farm. A year-long atmospheric LES driven with large-scale boundary conditions from the ERA5 [5] according to a setting described in [4] has been performed. The simulation domain covers $76.8 \mathrm{~km}$ by $86.4 \mathrm{~km}$ and is $4 \mathrm{~km}$ high with a horizontal resolution of $100 \mathrm{~m}$ and a vertical resolution of $25 \mathrm{~m}$. A total of 275 turbines of the IEA-15 MW reference turbine [6] type was included on the location of the IJmuiden-Ver wind farm zone that is currently being prepared for tendering. Large-scale wind fields as well as detailed LES wind fields are shown in figure 1 for a day with strong wake effects. A time series of 8 days of the power production and wind speed per turbine are shown in figure 2. The figure exposes how difficult it is to predict wind farm production: huge swings in power output over short time intervals, a spread of more than $50 \%$ between first row and fully waked turbines inside the farm on a given time, days of practically zero production followed by full $4 \mathrm{GW}$ production. To put these numbers into perspective: the average Dutch electricity demand is around $12 \mathrm{GW}$ and can be as low a $8 \mathrm{GW}$. This single large wind farm will thus intermittently cover between $0 \%$ and $50 \%$ of the total demand. Note how small free stream wind speed variations (between $8 \mathrm{~m} / \mathrm{s}$ and $10 \mathrm{~m} / \mathrm{s}$ ) lead to differences in power output of almost $80 \%$.

Why is it that wake effects inside the wind farm vary so much from time to time? Besides the dependence on wind speed (through the power curve) and wind direction (in relation to turbine arrangements), the vertical structure of the atmosphere is an important factor. To illustrate this, consider the vertical profiles of temperature and the wind speed deficit (reduction with respect to the free-stream wind speed) in the middle of the wind farm as shown in figure 3.

During the first few days, the atmosphere is well mixed over at least the first $500 \mathrm{~m}$ of the boundary layer. Around May $11^{\text {th }}$, a strong vertical temperature gradient develops, indicating a stable atmosphere in which turbulent mixing is suppressed. The wind speed decreases strongly around the rotor heights since momentum is extracted, but there is little turbulent entrainment from higher layers. Above the wind farm an increase in wind speed is visible, much like the acceleration of a stratified fluid over a small hill or obstacle.

\section{Global blockage and far wake effects}

A phenomenon that currently receives a lot of attention in the wind industry is global blockage [7]. Loosely speaking, global blockage refers to the combined induction effect that a wind farm as a whole exerts on the flow upstream of the wind farm. This phenomenon can be considered as a manifestation of a gravity wave, and can be understood by analogy with an object in a stream of water: through pressure forces, the fluid upstream will "feel" the obstacle and will flow around it. A deceleration in the streamwise direction combined with wave-like phenomena are the result of this balance between pressure and buoyancy forces. Upon close inspection, figure 1 shows some signatures of global blockage: on the east (right) side of the wind farm a prolonged region of lower wind speed exists. On the west (left) of the wind farm, a wave-like pattern can be observed. The vertical profile of the

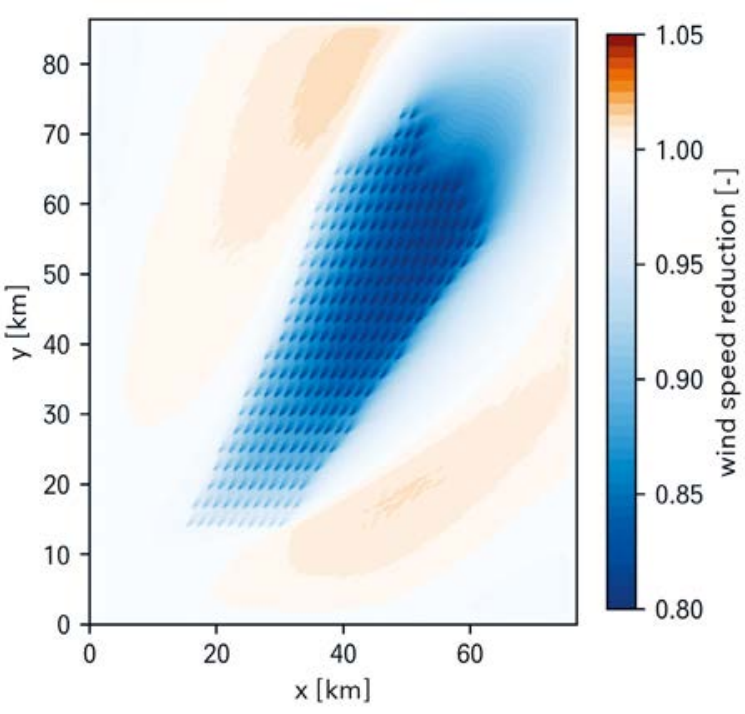

\FIG.4:Composite plot forall south-westerly wind directions of the velocity deficit. 
southern met-mast shows a reduction in wind speed compared to the free-stream wind. A yearlong LES run provides interesting possibilities to look at flow statistics conditioned on atmospheric circumstances. For example, one could take the average wind speed deficit for all situations with south-westerly winds, shown in figure 4 . Apart from the stronger wake effects deeper in the wind farm, a noteworthy feature is the acceleration of the flow alongside the wind farm. Though consistent with the interpretation of blockage as gravity waves, the existence of such accelerations is yet to be confirmed by measurement campaigns.

As favourable locations for wind energy become more crowded, the inter-wind-farm wake effects will become more prominent. Observations of wake effects extending for tens of kilometres, thereby affecting down-stream wind farms, have already been reported. Figure 4 shows that such effects are also present in the LES runs of the $4 \mathrm{GW}$ wind farm. Turbulent mixing, strongly related to atmospheric stability, will determine how fast high-momentum air from higher altitudes is mixed into the wake zone. The wind energy potential of a certain region will thus ultimately be dictated by the balance between extraction of kinetic energy (converted to electricity) and the turbulent flux of kinetic energy from above.

\section{Prospects and challenges}

Despite the scientific advances that enable us to get a better grip on wind farm flow physics, some open questions remain, many of which fall in the category of wind-farm atmosphere interactions. Innovative wind farm control strategies are aimed at enhancing mixing, for example in the helix approach [8] where individual turbine blades are manipulated in a way that creates a helical wake zone. An unanswered question is what the limits to vertical entrainment of momentum are under control strategies aimed at increasing turbulent mixing.

Turbulence is also a critical factor in wind turbine design. Today's design practices are largely based on synthetic turbulence fields that respect some but not all turbulence characteristics. A more natural approach would be to embed an aero-elastic model directly into an LES - an avenue that is becoming feasible with today's computer power.

In the end, the entire oceanic and meteorological environment of a wind farm matters for its design. Waves, currents, turbulence, extreme gusts, icing, impacts of hydrometeors (raindrops) on blades: once built, the turbines need to withstand it all. A better understanding of these processes will bring down capital and operational costs of wind energy.

The fact that renewable energy sources are so intimately coupled to the atmosphere could well lead to a prosperous era for the atmospheric sciences. The need to transform our energy system is bringing this field to the forefront of our new industrial revolution and puts atmospheric physics for wind farms firmly on the scientific agenda.

\section{About the author

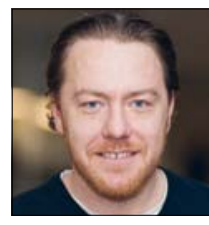 \\ R.A. Verzijlbergh is Associate profes- sor at Delft University of Technology, faculty of Technology, Policy and Management. He is Director of oper- ations at Whiffle Weather Forecasting.}

\section{References}

[1] P. Veers et al., Science 366, 6464 (2019)

[2] J. Schalkwijk, H.J.J. Jonker, A.P. Siebesma and E. Van Meijgaard, Bulletin of the American Meteorological Society 96(5), 715 (2015)

[3] Jeremy A. Sauer and Domingo Muñoz-Esparza, Journal of Advances in Modeling Earth Systems, 12(11), (2020).

[4] C. Gilbert, J.W. Messner, P. Pinson, P.-J. Trombe, R. Verzijlbergh, P. van Dorp, and H. Jonker. Wind Energy 23(4), (2020)

[5] H. Hersbach et al., Quarterly Journal of the Royal Meteorological Society 146(730), 1999, (2020)

[6] E. Gaertner et al., Technical report, International Energy Agency, (2020)

[7] J. Bleeg, M. Purcell, R. Ruisi and E. Traiger, Energies 11(6) (2018),

[8] J.A. Frederik, B.M. Doekemeijer, S.P. Mulders and J. Willem van Wingerden, Wind Energy 23(8), 1739 (2020)

\section{NEW EPS HISTORIC SITES}

Check them out on page 43!

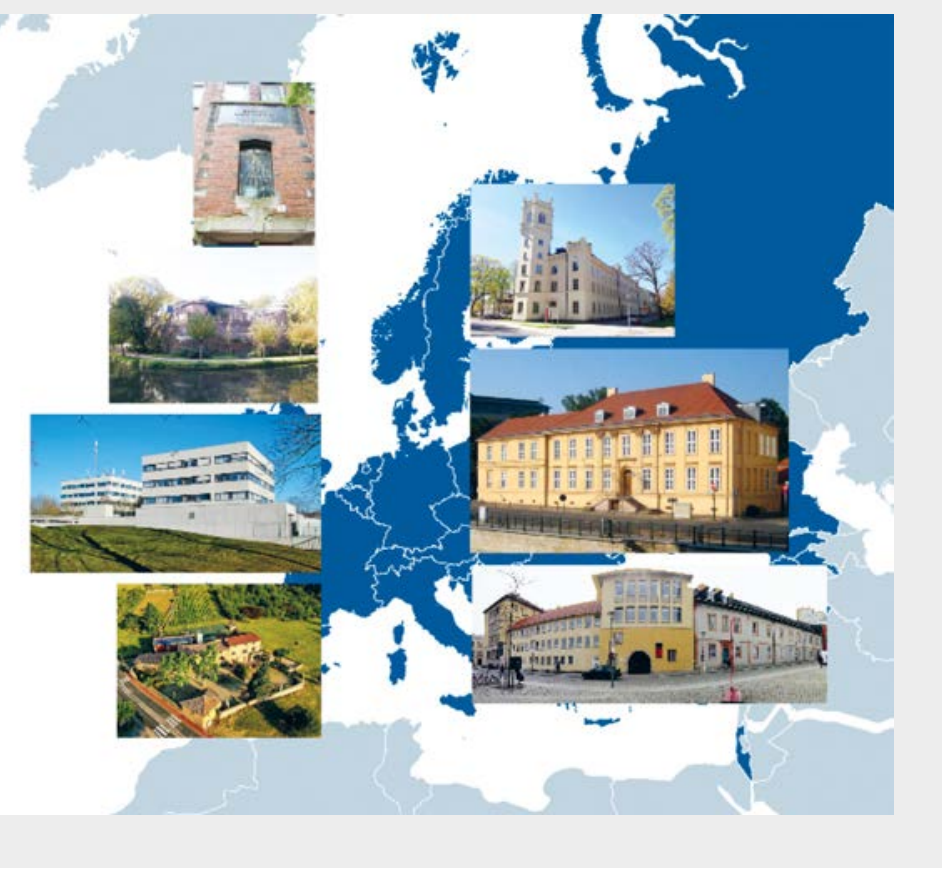

